\title{
Fractional biharmonic operator equation model for arbitrary frequency-dependent scattering attenuation in acoustic wave propagation
}

\author{
Wen Chen, ${ }^{1, a)}$ Jun Fang, ${ }^{1, b)}$ Guofei Pang, ${ }^{2}$ and Sverre Holm ${ }^{3}$ \\ ${ }^{1}$ State Key Laboratory of Hydrology-Water Resources and Hydraulic Engineering, Institute of Soft Matter \\ Mechanics, College of Mechanics and Materials, Hohai University, Fuchengxi Road 8, Jiangning District, \\ Nanjing, Jiangsu Province 211100, People's Republic of China \\ ${ }^{2}$ Beijing Computational Science Research Center, No. 10 East Xibeiwang Road, Haidian District, \\ Beijing 100193, People's Republic of China \\ ${ }^{3}$ Department of Informatics, University of Oslo, P. O. Box 1080, NO-0316 Oslo, Norway
}

(Received 17 July 2016; revised 9 November 2016; accepted 28 December 2016; published online 17 January 2017)

\begin{abstract}
This paper proposes a fractional biharmonic operator equation model in the time-space domain to describe scattering attenuation of acoustic waves in heterogeneous media. Compared with the existing models, the proposed fractional model is able to describe arbitrary frequency-dependent scattering attenuation, which typically obeys an empirical power law with an exponent ranging from 0 to 4. In stark contrast to an extensive and rapidly increasing application of the fractional derivative models for wave absorption attenuation in the literature, little has been reported on frequencydependent scattering attenuation. This is largely because the order of the fractional Laplacian is from 0 to 2 and is infeasible for scattering attenuation. In this study, the definition of the fractional biharmonic operator in space with an order varying from 0 to 4 is proposed, as well as a fractional biharmonic operator equation model of scattering attenuation which is consistent with arbitrary frequency power-law dependency and obeys the causal relation under the smallness approximation. Finally, the correlation between the fractional order and the ratio of wavelength to the diameter of the scattering heterogeneity is investigated and an expression on exponential form is also provided. (C) 2017 Acoustical Society of America. [http://dx.doi.org/10.1121/1.4973865]
\end{abstract}

$[\mathrm{LH}]$

Pages: 244-253

\section{INTRODUCTION}

Acoustic wave attenuation refers to energy loss during propagation in media. Since it reflects the inherent media characteristics in which the wave propagates, the acoustic wave attenuation has been a main concern in a wide variety of scientific and engineering fields, such as diagnostic ultrasound imaging, ${ }^{1,2}$ exploration seismology, ${ }^{3}$ and ultrasonic non-destructive inspection, ${ }^{4,5}$ just to mention a few.

There are many factors that cause acoustic wave attenuation, including geometry attenuation (like spherical spreading), absorption attenuation (intrinsic attenuation), and scattering attenuation. 3,6 The geometry attenuation is caused by energy dispersing during propagation and is independent of frequency. The absorptive attenuation is due to media viscosity, while the scattering attenuation is caused by media heterogeneity. Experimental results and field observations indicate that scattering attenuation plays a key role in acoustic wave propagation in some heterogeneous porous media such as rock and concrete ${ }^{7}$ and has great utility in engineering and scientific research. However, modeling such scattering behavior in complex media is not a simple task, due to its frequency-dependent behavior.

\footnotetext{
a)Electronic mail: chenwen@hhu.edu.cn

b) Current address: Department of Informatics, University of Oslo, P. O. Box 1080, NO-0316 Oslo, Norway.
}

Based on observations and experiments, some empirical regularities of scattering attenuation are found. When many anomalies are present in the medium, wave propagation resembles a random walk and scattering attenuation takes place. It actually is a kind of material interior geometric effect by which the energy is redistributed in time and space. $^{8}$ In order to describe the energy loss caused by scattering attenuation, the scattering coefficient $\alpha$ is usually used to represent the partial loss of energy from primary waves by scattering per unit travel distance. It is widely observed that the scattering attenuation in the medium obeys an empirical formula, ${ }^{9,10}$

$$
E=E_{0} e^{-\alpha x}
$$

where $E$ denotes the amplitude of an acoustic field variable such as velocity or pressure and $x$ is the wave's propagation distance. If the size of the scattering heterogeneity $d$ is much greater than the wavelength $\lambda(\lambda \ll d)$, the attenuation coefficient $\alpha$ is proportional to $\alpha \propto d^{-1}$, i.e., diffusion scattering. If the wavelength is much larger than the size of anomalies $(\lambda \gg d)$, the wave propagates through an equivalent continuum which combines the properties of the host medium and the inclusions. In this case, the attenuation coefficient varies proportionally to $\alpha \propto d^{3} \omega^{4}$, i.e., Rayleigh scattering. When the wavelength approaches the size of the inclusions $(\lambda \approx d)$, phase scattering takes place and the attenuation 
coefficient varies proportionally to $\alpha \propto d \omega^{2}$, i.e., Stochastic/ Mie scattering. ${ }^{11}$ Generally speaking, the attenuation coefficient $\alpha$ that results from elastic scattering depends on the ratio of acoustic wavelength $\lambda$ to the diameter $d$ of the scattering heterogeneity and can be described as ${ }^{12}$

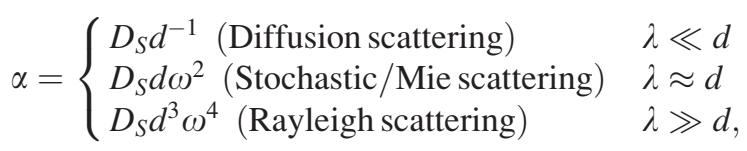

where $D_{S}$ is media-specific attenuation parameter obtained through a fitting of measured data. The wavelength can be written as $\lambda=c_{0} / \omega$ where $c_{0}$ is the wave velocity. Thus, the attenuation coefficient $\alpha(\omega)$ can be described with an empirical power law,

$$
\alpha(\omega)=D_{S} d^{s-1} \omega^{s}=\alpha_{0} \omega^{s}, \quad 0 \leq s \leq 4,
$$

where $\alpha_{0}=D_{S} d^{s-1}$ is the attenuation parameter related to scattering heterogeneity $d$. In special cases $s=0,2,4$, the classical model Eq. (2) is obtained. It is noted that the index $s$ can also be some non-integer values between 0 to 4 in certain case, for example, in seismic wave scattering attenuation. ${ }^{13,14}$ Thus, it is reasonable to assume that $s$ can be any real number between 0 and 4 . An expression that describes how the index $s$ changes with other variables will be discussed later in Sec. III. Above all, it remains a challenging open problem in timespace partial differential equation modeling to describe arbitrarily frequency-dependent scattering attenuation typically obeying an empirical power law as Eq. (3).

In recent years, the fractional derivative models have been developed to describe absorption attenuation in complex materials, such as anomalous diffusion, ${ }^{15,16}$ viscoelastic damping, ${ }^{17}$ and frequency-dependent dissipative wave propagation. ${ }^{18,19}$ With fractional derivative terms underlying the memory and long-range interaction, these models successfully describe the frequency-dependent behavior. ${ }^{20}$ Szabo derived a causal convolution operator for the lossy wave equation that accounted for power law absorption with a non-integer frequency dependence. ${ }^{21}$ This wave equation was later rewritten as a positive time-fractional derivative wave equation by Chen and Holm, ${ }^{18}$ and then developed by Kelly et $a l .^{22}$ However, the time-only operation in the above models is not feasible in the general case where the interaction between two oppositely traveling sound waves cannot be neglected, ${ }^{23}$ and they were derived mostly due to their ease of analysis. Therefore, Chen and Holm introduced the fractional Laplacian wave equation including a spatial fractional derivative operator ${ }^{19}$ based on Stokes' wave equation. Later, Treeby and Cox proposed an enhanced, causal fractional Laplacian wave equation which can describe power law absorption and dispersion for acoustic propagation. ${ }^{24}$ In contrast to these phenomenological models, another kind of fractional wave equations proposed by Holm et al. are derived differently from the viscoelastic constitutive equation, which is causal for any frequency and fulfills the Kramers-Kronig relation. ${ }^{25,26}$ However, little of past work concerns scattering attenuation in acoustic wave propagation. Besides the different physical mechanisms of absorption attenuation and scattering attenuation as discussed above, this is largely because the conventional spatial fractional Laplacian ${ }^{19}$ with an order from 0 to 2 cannot describe the scattering attenuation where the empirical scattering power law has an exponent ranging from 0 to 4 .

Based on the above-mentioned studies, this paper proposes new fractional derivative models to describe the acoustic wave scattering attenuation in heterogeneous media. To solve the problem, we introduce a new definition of the space-fractional derivative with an order from 0 to 4 , called the fractional biharmonic operator. And then the new fractional operator is applied to the scattering attenuation wave equation modeling.

Under the common smallness approximation, where attenuation is assumed to be much smaller than the wave number, the proposed fractional biharmonic operator equation model of acoustic scattering is found consistent with arbitrary frequency power-law dependency and obeys the causal relation. Furthermore, the correlation between the fractional order and the ratio of wavelength to diameter of the scattering heterogeneity is investigated and a possible form of expression is given. In order to verify this relationship, an existing scattering theory which has been demonstrated by experimental data is mentioned and then compared with our corresponding conclusions.

The rest of this paper is organized as follows. In Sec. II, the different types of fractional equation models for wave scattering attenuation are discussed so as to propose a new fractional biharmonic operator equation model. Then a physical interpretation and expression on the order of the fractional biharmonic operator is presented in Sec. III. The conclusions are presented in Sec. IV. In the Appendix, the existing definitions of the fractional Laplacian and our new definition of the fractional biharmonic operator are introduced.

\section{FRACTIONAL BIHARMONIC OPERATOR EQUATION MODEL OF WAVE SCATTERING ATTENUATION}

An attenuation wave equation can generally be described as ${ }^{24}$

$$
\Delta p-\frac{1}{c_{0}^{2}} \frac{\partial^{2} p}{\partial t^{2}}+\chi(p)=0
$$

where $p$ is the pressure of the acoustic wave, $c_{0}$ represents the phase velocity at a reference frequency, and $\chi$ denotes the attenuation term in form of time or space derivative operator. According to the existing equations of fractional order acoustic absorption attenuation models, it is reasonable to assume that the attenuation term of wave scattering is also a fractional mixed partial derivative in space and time which is suitable for description of the frequency-dependent power law attenuation.

\section{A. Scattering wave equation with a monomial attenuation term of time-space fractional derivative}

A monomial attenuation term in form of time and space fractional derivative operator can be described as 


$$
\chi(p)=C \frac{\partial^{a}}{\partial t^{a}}(-\Delta)^{b / 2} p
$$

where $C$ is the coefficient, $(-\Delta)^{b / 2}$ means the fractional biharmonic operator defined by Eq. (A13), and $\partial^{a} / \partial t^{a}$ is the time-fractional derivative operator defined by Eq. (A1).

In order to determine the coefficients and parameters and to verify that our scattering attenuation acoustic wave equation reflects the frequency-dependent power law attenuation of Eq. (3), the frequency-domain Fourier analysis is given below.

Applying the time and space Fourier transforms to Eqs. (4) and (5), in consideration of Eqs. (A2) and (A14), the frequency domain version of Eq. (4) combined with Eq. (5) is $^{19,27}$

$$
-k^{2}+\frac{\omega^{2}}{c_{0}^{2}}+C(i \omega)^{a} k^{b}=0
$$

where $k$ and $\omega$ are wave number and frequency variables, respectively. Splitting the wave number into real and imaginary parts where $k=\beta+i \alpha$, it is straightforward to have

$$
\begin{gathered}
-\beta^{2}+\alpha^{2}-i 2 \alpha \beta+\frac{\omega^{2}}{c_{0}^{2}}+C \cos \left(\frac{a \pi}{2}\right) \omega^{a} k^{b} \\
+i C \sin \left(\frac{a \pi}{2}\right) \omega^{a} k^{b}=0 .
\end{gathered}
$$

Here $\beta=\omega / c_{p}$ encapsulates the propagating part of the waves and $\alpha$ encapsulates the scattering attenuation. Though the complex wave number is usually used to describe wave absorption attenuation through lossy materials, it is reasonable to describe the wave scattering in the same way because it also results in both attenuation and dispersion. ${ }^{28,29}$

Separating the above equation, the real and imaginary parts are given by

$$
\left\{\begin{array}{l}
\operatorname{Re}: \alpha^{2}-\beta^{2}+\frac{\omega^{2}}{c_{0}^{2}}+C \cos (a \pi / 2) \omega^{a} k^{b}=0 \\
\operatorname{Im}: 2 \beta \alpha+C \sin (a \pi / 2) \omega^{a} k^{b}=0 .
\end{array}\right.
$$

With the conservative value under the smallness approximation, where attenuation is assumed to be much smaller than the wave number, ${ }^{30,31}$ a relationship is given by

$$
k \approx \beta=\omega / c_{p}=\omega / c_{0} .
$$

Simplifying the imaginary component of Eq. (8) then yields

$$
\alpha=-\frac{C \sin (a \pi / 2) \beta^{b}}{2 \beta} \omega^{a}=-\frac{C \sin (a \pi / 2)}{2 c_{0}^{b-1}} \omega^{a+b-1} .
$$

It is noted that Eq. (10) is consistent with the empirical power law Eq. (3), namely,

$$
\left\{\begin{array}{l}
a+b=s+1 \\
C=-\frac{2 a_{0} d^{s-1} c_{0}^{s-a}}{\sin (a \pi / 2)}=-\frac{2 \alpha_{0} c_{0}^{s-a}}{\sin (a \pi / 2)} .
\end{array}\right.
$$

In terms of the relationship formulas (11), if the time and space fractional orders and the coefficient $C$ in Eq. (5) agree with Eq. (11), then the fractional wave scattering attenuation equation for the power-law frequency-dependent attenuation in heterogeneous media is given.

In the simplest case, let $a=1, b=s$, the scattering attenuation equation is stated as

$$
\Delta p-\frac{1}{c_{0}^{2}} \frac{\partial^{2} p}{\partial t^{2}}-2 \alpha_{0} c_{0}^{s-1} \frac{\partial}{\partial t}(-\Delta)^{s / 2} p=0,0 \leq s \leq 4,
$$

which appears similar to the fractional Laplacian equation of dissipative acoustic wave proposed by Chen and Holm. ${ }^{19}$ The difference is that the order of the fractional Laplacian is from 0 to 2 to describe wave absorption attenuation while the order of the fractional biharmonic operator here is from 0 to 4. However, Treeby and Cox found that this form of the wave equation exhibit the desired power law attenuation but is non-causal. ${ }^{24}$

In wave equation models, causality must be considered. The propagation of a sound wave through a heterogeneous medium is intrinsically linked with dispersion, a dependence of the phase speed on frequency. ${ }^{24}$

In order to solve this problem, simplifying the real component of Eq. (8) under the smallness assumption $\alpha \ll \beta$, Eqs. (9) and (11) then yield

$$
\begin{aligned}
\beta & =\sqrt{\alpha^{2}+\frac{\omega^{2}}{c_{0}^{2}}+C \cos \left(\frac{a \pi}{2}\right) \omega^{a} k^{b}} \\
& \approx \frac{\omega}{c_{0}} \sqrt{1+C \cos \left(\frac{a \pi}{2}\right) c_{0}^{a+1-s} \omega^{s-1}} .
\end{aligned}
$$

Applying Taylor expansion and reserving the first two terms $\left[C \cos (a \pi / 2) c_{0}^{a+1-s} \omega^{s-1} \ll 1\right]$ then gives

$$
\begin{aligned}
\beta & =\frac{\omega}{c_{0}}+\frac{C}{2 c_{0}^{s-a}} \cos \left(\frac{a \pi}{2}\right) \omega^{s} \\
& =\frac{\omega}{c_{0}}+\alpha_{0} \tan \left(\frac{(a-1) \pi}{2}\right) \omega^{s},
\end{aligned}
$$

where the coefficient $C$ is given by Eq. (11).

Based on the causal relation, Horton ${ }^{32,33}$ published a set of dispersion relations of power-law attenuation stated as follows

$$
\beta=\frac{\omega}{c_{0}}+\alpha_{0} \tan \left(\frac{s \pi}{2}\right) \omega^{s}
$$

Here $0<s<1$. Some reports suggest that this causal relation is also valid for $1<s<3 .{ }^{34,35}$ Analogously, it can be reasonably inferred from the literature that Eq. (15) is valid for $0 \leq s \leq 4$ and $s \neq 1,3$ (an alternate expression is also available for $s=1$ or 3$).{ }^{34}$

Comparing Eq. (14) with Eq. (15) then yields

$$
a=s+1-2 n \text {. }
$$


Here $n$ is an integer. If the space fractional order $b=2 n$ assuming $n=0,1,2$, the scattering attenuation equation can obey the causal relation and satisfy the empirical power law in the following expression

$$
\begin{aligned}
& \Delta p-\frac{1}{c_{0}^{2}} \frac{\partial^{2} p}{\partial t^{2}}-\frac{2 \alpha_{0} c_{0}^{2 n-1}}{\sin [(s+1-2 n) \pi / 2]} \frac{\partial^{s+1-2 n}}{\partial t^{s+1-2 n}}(-\Delta)^{n} p=0 \\
& 0 \leq s \leq 4, \quad s \neq 1,3 ; \quad n=0,1,2 .
\end{aligned}
$$

In the simplest expression, $n=0, a=s+1, b=0$, the equation can be reduced to

$$
\begin{aligned}
& \Delta p-\frac{1}{c_{0}^{2}} \frac{\partial^{2} p}{\partial t^{2}}-\frac{2 \alpha_{0}}{c_{0} \cos (s \pi / 2)} \frac{\partial^{s+1}}{\partial t^{s+1}} p=0, \\
& 0 \leq s \leq 4, \quad s \neq 1,3,
\end{aligned}
$$

which has a similar expression as the integro-differential operator equation proposed by $\mathrm{Szabo}^{21}$ for viscosity dissipation but the parameter has different physical significance and range. While $n=1, a=s-1, b=2$, the equation can be described as

$$
\begin{aligned}
& \Delta p-\frac{1}{c_{0}^{2}} \frac{\partial^{2} p}{\partial t^{2}}+\frac{2 \alpha_{0} c_{0}}{\cos (s \pi / 2)} \frac{\partial^{s-1}}{\partial t^{s-1}} \Delta p=0, \\
& 0 \leq s \leq 4, \quad s \neq 1,3
\end{aligned}
$$

which appears similar to the fractional derivative equation proposed by Caputo and Wismer ${ }^{36,37}$ but is physically different. It is worth mentioning that since the Caputo/Wismer equation is based on a causal constitutive equation, it is causal without the smallness approximation (valid for all frequencies). ${ }^{38}$
In general, a monomial attenuation term which obeys the causal relation and satisfies the empirical power law of scattering cannot be expressed only by the space fractional derivative operator. However, considering that scattering attenuation is caused by media heterogeneity, a space-fractional derivative is appropriate to characterize the scattering attenuation for the spatial non-locality and power law behavior. It is also noted that the scattering representation Eq. (19) involves the temporal derivative of 3 order when $s=4$. This could cause some problems in the solution of real-world problems. In Sec. II B, two terms for the fractional space derivative are therefore employed to represent scattering attenuation to avoid the high-order temporal derivative issue.

\section{B. Scattering wave equation with two terms of fractional space derivative}

The attenuation term in the scattering wave equation model should exhibit the correct power law and satisfy a causal dispersion relationship, under appropriate conditions. In this section, the two terms of scattering attenuation expression in Eq. (4) are given by

$$
\chi(p)=C_{1} \frac{\partial^{a}}{\partial t^{a}}(-\Delta)^{(b / 2)} p+C_{2} \frac{\partial^{p}}{\partial t^{p}}(-\Delta)^{(q / 2)} p
$$

where $C_{1}$ and $C_{2}$ are the undetermined coefficients. This form of attenuation (with $a=1$ and $p=0$, specially) was first proposed by Treeby and Cox to describe causal absorption attenuation. $^{24}$

Applying the time and space Fourier transforms to Eq. (20) and separating the frequency domain equation, the real and imaginary parts are given by

$$
\left\{\begin{array}{l}
\operatorname{Re}: \alpha^{2}-\beta^{2}+\frac{\omega^{2}}{c_{0}^{2}}+C_{1} \cos (a \pi / 2) \omega^{a} k^{b}+C_{2} \cos (p \pi / 2) \omega^{p} k^{q}=0 \\
\operatorname{Im}: 2 \beta \alpha+C_{1} \sin (a \pi / 2) \omega^{a} k^{b}+C_{2} \sin (p \pi / 2) \omega^{p} k^{q}=0 .
\end{array}\right.
$$

From the preceding analysis, it is evident that the real part of Eq. (21) corresponds to the encapsulated dispersion, while the imaginary part represents the scattering attenuation. Without loss of generality, one may assume that the first part of the attenuation term exhibits only the power law attenuation while the second part represents only the dispersion. Thus the coefficient $C_{1}$ of the first attenuation term will not appear in the real part of Eq. (21) otherwise it will affect the dispersion. Equally, the coefficient $C_{2}$ of the first attenuation term will not appear in the imaginary part of Eq. (21) otherwise it will affect the scattering attenuation. This assumption then gives

$$
\begin{cases}\cos (a \pi / 2)=0, & a=2 m+1 \\ \sin (p \pi / 2)=0, & p=2 n .\end{cases}
$$

Here $m, n$ are integers. Under the smallness approximation Eq. (9), simplifying the imaginary component of Eq. (21) then yields

$$
\alpha=(-1)^{m+1} \frac{C_{1} \beta^{b}}{2 \beta} \omega^{2 m+1}=(-1)^{m+1} \frac{C_{1}}{2 c_{0}^{b-1}} \omega^{2 m+b} .
$$

Comparing with the empirical power law Eq. (3) then gives

$$
\left\{\begin{array}{l}
b=s-2 m \\
C_{1}=(-1)^{m+1} 2 a_{0} d^{s-1} c_{0}^{b-1}=(-1)^{m+1} 2 \alpha_{0} c_{0}^{s-1-2 m} .
\end{array}\right.
$$

The real component of Eq. (21) can be simplified in the same way as in Sec. II A and becomes 


$$
\beta=\frac{\omega}{c_{0}}+(-1)^{n} \frac{C_{2}}{2 c_{0}^{q-1}} \omega^{2 n-1+q} .
$$

Taking in consideration the causal relation of Eq. (15) then yields

$$
\left\{\begin{array}{l}
q=s+1-2 n \\
C_{2}=(-1)^{n} 2 \alpha_{0} c_{0}^{s-2 n} \tan (s \pi / 2) .
\end{array}\right.
$$

In order to avoid making the time and space fractional operators with too large orders beyond the scope of our definitions, assume $m=0,1$ and $n=0,1$, then the fractional biharmonic operator equation model for acoustic wave scattering attenuation can generally be written as

$$
\begin{gathered}
\Delta p-\frac{1}{c_{0}^{2}} \frac{\partial^{2} p}{\partial t^{2}}+(-1)^{m+1} 2 \alpha_{0} c_{0}^{s-1-2 m} \frac{\partial^{2 m+1}}{\partial t^{2 m+1}}(-\Delta)^{[(s-2 m) / 2]} p \\
+(-1)^{n} 2 \alpha_{0} c_{0}^{s-2 n} \tan \left(\frac{s \pi}{2}\right) \frac{\partial^{2 n}}{\partial t^{2 n}}(-\Delta)^{[(s+1-2 n) / 2]} p=0 \\
0 \leq s \leq 4, \quad s \neq 1,3 ; \quad m=0,1 ; \quad n=0,1 .
\end{gathered}
$$

When $m=0, n=0$, the equation can be reduced to

$$
\begin{aligned}
& \Delta p-\frac{1}{c_{0}^{2}} \frac{\partial^{2} p}{\partial t^{2}}-2 \alpha_{0} c_{0}^{s-1} \frac{\partial}{\partial t}(-\Delta)^{(s / 2)} p+2 \alpha_{0} c_{0}^{s} \\
& \times \tan \left(\frac{s \pi}{2}\right)(-\Delta)^{[(s+1) / 2]} p=0, \\
& 0 \leq s \leq 4, \quad s \neq 1,3,
\end{aligned}
$$

which looks like the fractional derivative equation proposed by Treeby and $\mathrm{Cox}^{24}$ for frequency-dependent dissipative attenuation, but the index $s$ ranges from 0 to 2 in the latter. It is worth mentioning that this type of attenuation term might be infeasible for scattering attenuation when the fractional order $s$ approaches 4 and the second part of the attenuation term is over fourth-order spatial derivative operator. This is unphysical.

In the case of $m=0, n=1$, Eq. (27) can also be rewritten as

$$
\begin{aligned}
& \Delta p-\frac{1}{c_{0}^{2}} \frac{\partial^{2} p}{\partial t^{2}}-2 \alpha_{0} c_{0}^{s-1} \frac{\partial}{\partial t}(-\Delta)^{(s / 2)} p-2 \alpha_{0} c_{0}^{s-2} \\
& \quad \times \tan \left(\frac{s \pi}{2}\right) \frac{\partial^{2}}{\partial t^{2}}(-\Delta)^{[(s-1) / 2]} p=0, \\
& 0 \leq s \leq 4, \quad s \neq 1,3
\end{aligned}
$$

which appears similar to the fractional derivative equation proposed by $\mathrm{Pang}^{29}$ for frequency-dependent dissipative attenuation. The highest fractional order of the attenuation term in Eq. (29) is $s-1$ and less than 4 instead of $s+1$ in Eq. (28). However, when the fractional order $s<1$, the last operator of the attenuation term is the inverse operator of the fractional Laplacian which is defined by Eq. (A4). In this scenario, the equation can be rewritten as

$$
\begin{aligned}
& \Delta p-\frac{1}{c_{0}^{2}} \frac{\partial^{2} p}{\partial t^{2}}-2 \alpha_{0} c_{0}^{s-1} \frac{\partial}{\partial t}(-\Delta)^{(s / 2)} p-2 \alpha_{0} c_{0}^{s-2} \\
& \quad \times \tan \left(\frac{s \pi}{2}\right) \frac{\partial^{2}}{\partial t^{2}} I^{1-s} p=0 \\
& 0 \leq s \leq 4, \quad s \neq 1,3
\end{aligned}
$$

In addition, if $m=1, n=1$, Eq. (27) becomes

$$
\begin{aligned}
\Delta p & -\frac{1}{c_{0}^{2}} \frac{\partial^{2} p}{\partial t^{2}}+2 \alpha_{0} c_{0}^{s-3} \frac{\partial^{3}}{\partial t^{3}}(-\Delta)^{[(s-2) / 2]} p-2 \alpha_{0} c_{0}^{s-2} \\
& \times \tan \left(\frac{s \pi}{2}\right) \frac{\partial^{2}}{\partial t^{2}}(-\Delta)^{[(s-1) / 2]} p=0 \\
& 0 \leq s \leq 4, \quad s \neq 1,3
\end{aligned}
$$

when the order of the fractional biharmonic operator is less than 0 , it will be converted to the Riesz potential operator.

The above analysis shows that the wave equation of Eq. (27) is feasible to characterize power-law frequency-dependent attenuation in heterogeneous media which obeys the causal relation. In Sec. III, the fractional order is physically interpreted in terms of the wavelength and the diameter of the scattering heterogeneity.

\section{RELATIONSHIP FORMULA BETWEEN SCATTERING POWER LAW INDEX AND THE RATIO OF THE HETEROGENEITY DIAMETER TO ACOUSTIC WAVELENGTH}

As discussed above in Eq. (2), the order $s$ of the fractional derivative and scattering power law attenuation depends on the ratio of acoustic wavelength $\lambda$ to the diameter $d$ of the scattering heterogeneity. The correlation between the fractional order (scattering power law attenuation index) and the ratio can be described as

$$
s= \begin{cases}0, & \mu \rightarrow+\infty \\ 2, & \mu=b_{0} \\ 4, & \mu=0\end{cases}
$$

where $\mu=d / \lambda$ is the ratio of the diameter $d$ to acoustic wavelength $\lambda . b_{0}$ is the value of $\mu$ when the Stochastic/Mie scattering occurs. In some cases, ${ }^{9}$ it is approximately equal to $1 / 2 \pi$, while we take it as an undetermined parameter here in general. Taking into account that the value of $\mu$ is actually a continuous variable quantity, then the value of $s$ should also be a continuous variable instead of a "jump" variable. A possible relation consistent with Eq. (32) is an exponential expression given by

$$
s=4 e^{-\mu \ln 2 / b_{0}},
$$

when $\lambda \ll d, \mu \rightarrow+\infty$, formula (33) is reduced to $s=0$; when $\lambda=d / b_{0}, \mu=b_{0}$, formula (33) is reduced to $s=2$; when $\lambda \gg d, \mu=0$, formula (33) is reduced to $s=4$. The expression (33) is shown in Fig. 1.

However, it should be mentioned that expression (33) is only a possible relationship based on the existing scattering 


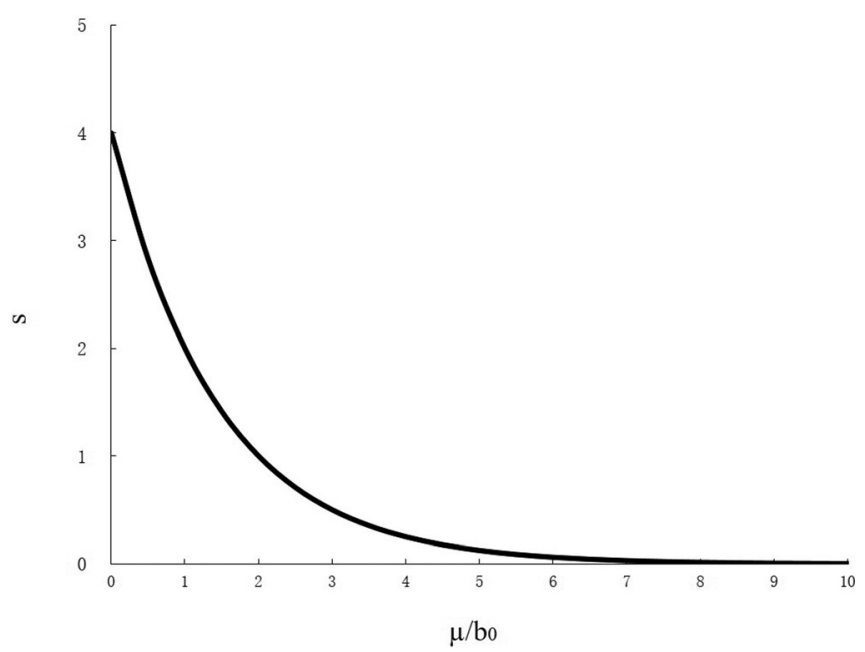

FIG. 1. The relation between the fractional order $s$ (power law scatter attenuation index) and the ratio $\mu$ of acoustic wavelength $\lambda$ to the diameter $d$ of the scattering heterogeneity described by formula (33). This figure shows an exponential curve which is possible to describe this relationship. The horizontal axis is normalized variables $\mu / b_{0}$.

theory such as Eq. (2), whether the expression is valid or not requires other theoretical or experimental verification.

In order to verify our conclusion, the scattering coefficient $\alpha$ must be studied. Substituting formula (33) into Eq. (3) and considering $\lambda=c_{0} / \omega$, the expression for the attenuation coefficient $\alpha$ as a function of $d$ and $\omega$ is as follows

$$
\alpha=D_{S} \omega(\omega d)^{4 \exp \left(-\omega d \ln 2 / b_{0} c_{0}\right)-1} .
$$

As a comparison, Blair ${ }^{14}$ has also shown that an acceptable frequency-dependent scattering attenuation coefficient $\alpha$ for elastic scattering can be cast in the general form

$$
\alpha=\frac{C_{S}}{d}\left(\frac{\omega}{\omega_{d}}\right)^{4}\left[1+\left(\frac{\omega}{\omega_{d}}\right)^{4}\right]^{-1}, \quad \omega_{d}=\frac{k_{s} c_{0}}{d},
$$

where $C_{S}$ and $k_{S}$ are constants, $c_{0}$ the wave velocity, $d$ the mean diameter of the scatterers, and $\omega_{d}$ the characteristic (constant) frequency, dependent only upon the scattering medium. Expression (35) is based on experimental data for seismic attenuation in rock and has also been observed in ultrasonic scattering in metals. It means that although the grain size in typical metals are significantly smaller than in most rock, scattering is a scale-independent phenomenon whose magnitude is determined by the ratio of wavelength to grain size. ${ }^{9}$ This conclusion is consistent with our scattering model.

To compare the two expressions for scattering coefficient and discuss the effect of the diameter $d$ and frequency $\omega$ on the attenuation coefficient $\alpha$, a graphical representation has been made to describe the relation between $\alpha$ and $d$ by formulas (34) and (35), respectively, under varying values of $\omega$ (5, 10,15 , and $20 \mathrm{~Hz}$ ). In order to simplify the calculation, we assume $D_{S}=C_{S}=1, c_{0}=1$, and take $k_{S}=0.23$ as proposed by Blair. When the parameter is $b_{0} \approx 1 / 4 \pi$, formula (34) is in good agreement with formula (35), as shown in Fig. 2.

From Fig. 2, it can also be observed that the crest region of $\alpha$ tends to be less acute when $\omega$ increases. It can also be seen that when the diameter $d$ of the scattering heterogeneity has a size approximately equal to the wavelength $\lambda(d \approx \pi \lambda)$, the attenuation coefficient $\alpha$ at a certain frequency $\omega$ reaches the maximum value. It means that similar size of the heterogeneity in the medium and wavelength causes stronger scattering attenuation. In other words, it means that the "Stochastic/Mie scattering" is the most significant type of scattering attenuation in the heterogeneous media. This conclusion is also consistent with the field and experiment observations.

\section{CONCLUSIONS}

This paper proposes a fractional biharmonic operator derivative equation model to describe the acoustic wave scattering attenuation in heterogeneous media. In

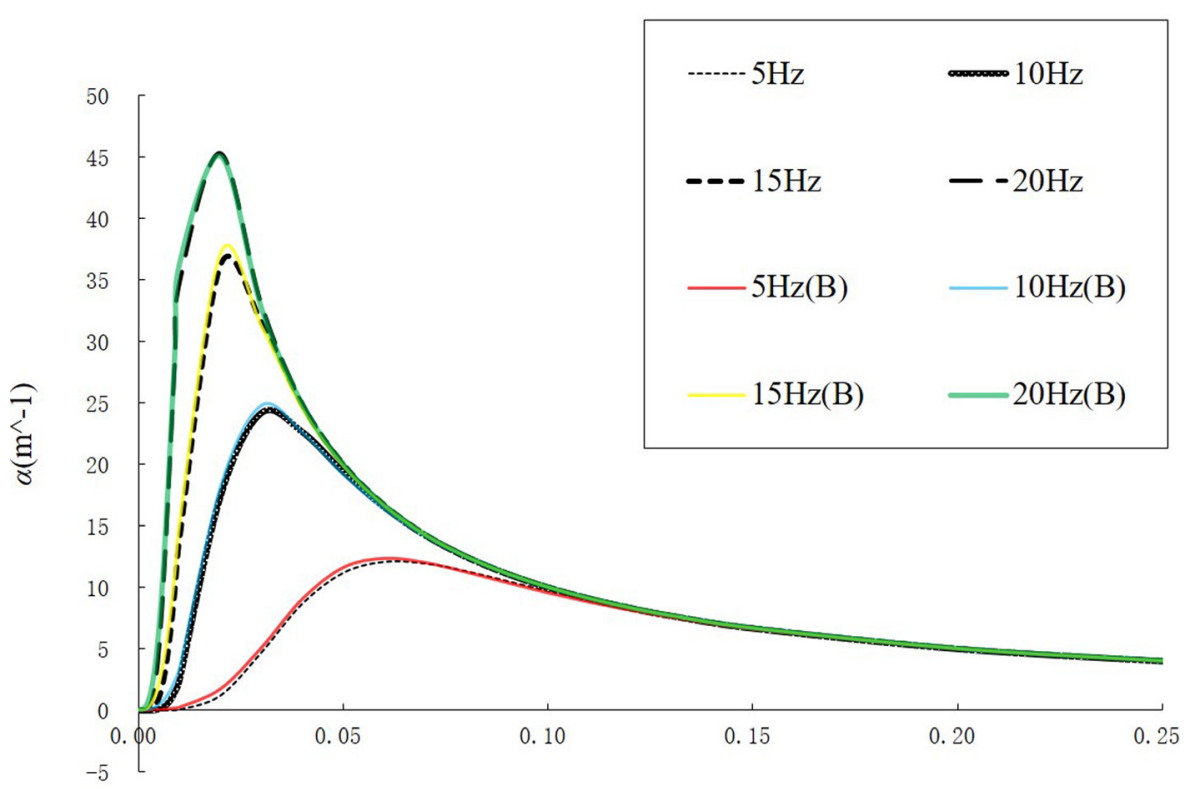

FIG. 2. (Color online) The relation between the scattering coefficient $\alpha$ and the diameter $d$ by formula (34) (black dotted line) and formula (35) (colorized curves) with varying frequency $\omega$. The curves of formula (34) are depicted by black dotted line versus the colorized curves of formula (35) proposed by Blair. The frequency $\omega$ varies from $5,10,15$ to $20 \mathrm{~Hz}$ with the parameters $D_{S}=C_{S}=1, c_{0}=1$, $k_{S}=0.23$, and $b_{0} \approx 1 / 4 \pi$. The figure shows that formula (34) is in good agreement with formula (35) in different cases.

$d(\mathrm{~m})$ 
consideration of the empirical power law with an exponent ranging from 0 to 4 , the definition of the space-fractional derivative, called fractional biharmonic operator, is introduced in the Appendix.

After the systematic analysis of the expression of scattering attenuation term, it is demonstrated that the wave Eqs. (17) and (27) are consistent with the empirical power law Eq. (3) and is capable of describing arbitrary scattering attenuation. While obeying the causal relation under the smallness approximation, it means that they can all describe both scattering attenuation and dispersion. This would be an important result since the assumption is usually made that there is no phase change. Specially, if the order of the fractional operators in expression (5) obeys $a=1$ and $b=s$, the scattering attenuation Eq. (12) is obtained without considering dispersion.

Moreover, the relationship between the fractional order (scattering attenuation power law index) and the ratio of wavelength to the diameter of the scattering heterogeneity is established. To verify this relationship, an existing scattering theory proposed by Blair which has been verified by experimental data is compared with our conclusions. The result shows that when the parameter of formula (34) $d_{0} \approx 1 / 4 \pi$ and the parameter of formula (35) $k_{S}=0.23$, the two curves are in good agreement. In another case, with $d_{0}=1 / 2 \pi$ and $k_{S}=0.4$, formula (34) is in good agreement with formula (35), too. In fact, we can come to the conclusion that the two expressions for scattering attenuation coefficient $\alpha$ are approximately equivalent while choosing the appropriate values of $d_{0}$ and $k_{S}$. Through the comparison, expression (33) which describes the relationship between the order of the fractional operator and the ratio of wavelength to the diameter of the scatters is then indirectly verified and appears valid for calculating the scattering attenuation.

\section{ACKNOWLEDGMENTS}

The work described in this paper was supported by the National Natural Science Foundation of China, the 111 project, and a Norwegian Government Scholarship to Chinese nationals from the Research Council of Norway.

\section{APPENDIX: DEFINITIONS OF THE FRACTIONAL DERIVATIVE}

\section{Existing definitions of the fractional derivative}

In order to prepare the introduction of the fractional biharmonic operator in space, this study begins with the existing definitions of time and space fractional derivatives. The Caputo's fractional derivative is a conventional time integro-differential operator and is written as follows.

\section{a. Definition A}

Caputo's time-fractional derivative with combination of the derivative and the convolution is given by ${ }^{39}$

$$
\begin{aligned}
\frac{\partial^{s}}{\partial t^{s}} \varphi(t)= & \frac{1}{\Gamma(n-s)} \int_{-\infty}^{t}(t-\tau)^{n-s-1} \varphi^{(n)}(\tau) d \tau \\
& n-1<s<n
\end{aligned}
$$

where $\Gamma(\cdot)$ denotes Euler's gamma function, $\varphi(t)$ must be $n$ order differentiable. In addition, the integer derivative of $\varphi(t)$ must be given at the initial instance, for example, when $n=1$ and the initial time equals $0, \varphi(0)$ and $\varphi^{\prime}(0)$ must been given as the initial conditions. The Riemann-Liouville definition of the fractional derivative can also be written in a similar form but the derivative is taken after the convolution. The Caputo type definition is the regularized form of the Riemann-Liouville type. The Fourier transform of the timefractional derivative has been given by Bagley and Torvik ${ }^{40}$

$$
F\left\{\frac{\partial^{s}}{\partial t^{s}} \varphi(t), \omega\right\}=(i \omega)^{s} \Phi(\omega) .
$$

The fractional Laplacian $(-\Delta)_{*}^{s / 2}(0<s \leq 2)$ is a spatial integro-differential operator and can describe the spatial non-locality and power law behavior of scientific and engineering problems. A variety of definitions of fractional Laplacian have been proposed in recent years. One of them is introduced by Chen and Holm. ${ }^{19}$ They used the Riesz potential $^{41}$ and the fractional integral in space via the Green second identity to propose an explicit integral expression of fractional Laplacian, which naturally includes the boundary conditions.

\section{b. Definition B}

Fractional operator as a composition of Riesz potentials and Laplacian ${ }^{19,42}$ is given by

$$
\begin{aligned}
(-\Delta)_{*}^{s / 2} \varphi(x)= & \left\{\begin{array}{l}
-\Delta\left[I_{d}^{2-s} \varphi(x)\right](\text { Riemann-Liouville-type }) \\
I_{d}^{2-s}[-\Delta \varphi(x)](\text { Caputo-type })
\end{array}\right. \\
& 0<s \leq 2
\end{aligned}
$$

where the Riesz potential operator of order $s$ of $d$ dimensions is defined by ${ }^{43,44}$

$$
\begin{aligned}
I_{d}^{s} \varphi(x)= & \frac{\Gamma[(d-s) / 2]}{\pi^{s / 2} 2^{s} \Gamma(s / 2)} \int_{\Omega} \frac{\varphi(\xi)}{\|x-\xi\|^{d-s}} d \Omega(\xi), \\
& 0<s<2
\end{aligned}
$$

and where $\Omega$ is the integral domain. The definition is used in modeling acoustic dissipation in human tissue. ${ }^{19}$

\section{New definition of factional biharmonic operator}

However, the fractional Laplacian Eq. (A3) with an order from 0 to 2 is not able to describe the empirical power law as Eq. (3) with an exponent ranging from 0 to 4 . This section introduces a new spatial integro-differential operator $(-\Delta)_{* *}^{s / 2}(2<s \leq 4)$, called fractional biharmonic operator. The definition is follows. 


\section{a. Definition C}

The fractional biharmonic operator as a composition of the Riesz potential and the biharmonic operator is given by

$$
\begin{aligned}
(-\Delta)_{* *}^{s / 2} \varphi(x)= & \left\{\begin{array}{l}
\Delta \Delta\left[I_{d}^{4-s} \varphi(x)\right](\text { Riemann-Liouville-type }) \\
I_{d}^{4-s}[\Delta \Delta \varphi(x)](\text { Caputo-type }),
\end{array}\right. \\
& 2<s \leq 4,
\end{aligned}
$$

the order $((4-s) \in(0,2])$ of Riesz potential operator is consistent with that in Eq. (A4). Although the current definitions of the fractional derivative with an order exceeding 2 are rare, Buckingham suggested that there is no upper or lower limit on the value that $s$ may take. ${ }^{45}$

It is known that the Laplacian operator has the radial basis function expression

$$
\Delta \varphi(x)=\frac{d^{2} \varphi}{d r^{2}}+\frac{d-1}{r} \frac{d \varphi}{d r}
$$

where $r=\|x-\xi\|$ denotes Euclidean distance. Equation (A5) can then be reduced to

$$
\begin{aligned}
\Delta \Delta\left[I_{d}^{4-s} \varphi(x)\right] & =\frac{\Gamma[(d-4+s) / 2]}{\pi^{4-s / 2} 2^{4-s} \Gamma[(4-s) / 2]} \Delta \Delta \int_{\Omega} \frac{\varphi(\xi)}{\|x-\xi\|^{d+s-4}} d \Omega(\xi) \\
& =\frac{(s+d-4)(s-2)(s+d-2) s \Gamma[(d-4+s) / 2]}{\pi^{(4-s) / 2} 2^{4-s} \Gamma[(4-s) / 2]} \int_{\Omega} \frac{\varphi(\xi)}{\|x-\xi\|^{d+s}} d \Omega(\xi), \\
I_{d}^{4-s}[\Delta \Delta \varphi(x)] & =\frac{\Gamma[(d-4+s) / 2]}{\pi^{(4-s) / 2} 2^{4-s} \Gamma[(4-s) / 2]} \int_{\Omega} \frac{\Delta \Delta \varphi(\xi)}{\|x-\xi\|^{d+s-4}} d \Omega(\xi) .
\end{aligned}
$$

It is noted that Eq. (A7) encounters the detrimental hypersingularity, which means the singularity order $d+s$ is larger than the topological dimension $d$, while the definition Eq. (A8) has a weak singularity of order $d+s-4$ compared with the hypersingularity of order $d+s$ in Eq. (A7). The Green's second identity is useful to connect Eqs. (A7) and (A8), ${ }^{19}$

$$
\begin{aligned}
I_{d}^{4-s}[\Delta \Delta \varphi(x)]= & \frac{(s+d-4)(s-2) \Gamma[(d-4+s) / 2]}{\pi^{(4-s) / 2} 2^{4-s} \Gamma[(4-s) / 2]} \int_{\Omega} \frac{\Delta \varphi(\xi)}{\|x-\xi\|^{d+s-2}} d \Omega(\xi) \\
& +h \int_{S}\left[\Delta \varphi(\xi) \frac{\partial}{\partial n}\left(\frac{1}{\|x-\xi\|^{d+s-4}}\right)-\frac{1}{\|x-\xi\|^{d+s-4}} \frac{\partial}{\partial n} \Delta \varphi(\xi)\right] d S(\xi) \\
= & \frac{(s+d-4)(s-2)(s+d-2) s \Gamma[(d-4+s) / 2]}{\pi^{(4-s) / 2} 2^{4-s} \Gamma[(4-s) / 2]} \int_{\Omega} \frac{\varphi(\xi)}{\|x-\xi\|^{d+s}} d \Omega(\xi) \\
& +g \int_{S}\left[\varphi(\xi) \frac{\partial}{\partial n}\left(\frac{1}{\|x-\xi\|^{d+s-2}}\right)-\frac{1}{\|x-\xi\|^{d+s-2}} \frac{\partial \varphi(\xi)}{\partial n}\right] d S(\xi) \\
& +h \int_{S}\left[C(\xi) \frac{\partial}{\partial n}\left(\frac{1}{\|x-\xi\|^{d+s-4}}\right)-\frac{D(\xi)}{\|x-\xi\|^{d+s-4}}\right] d S(\xi) \\
= & \Delta \Delta\left[I_{d}^{4-s} \varphi(x)\right]+g \int_{S}\left[E(\xi) \frac{\partial}{\partial n}\left(\frac{1}{\|x-\xi\|^{d+s-2}}\right)-\frac{F(\xi)}{\|x-\xi\|^{d+s-2}}\right] d S(\xi) \\
& +h \int_{S}\left[C(\xi) \frac{\partial}{\partial n}\left(\frac{1}{\|x-\xi\|^{d+s-4}}\right)-\frac{D(\xi)}{\|x-\xi\|^{d+s-4}}\right] d S(\xi),
\end{aligned}
$$

where $S$ represents the surface of the domain, and $n$ is the unit outward normal. Let

$$
\begin{aligned}
& \left.\Delta \varphi(x)\right|_{x \in S}=C(x),\left.\quad \varphi(x)\right|_{x \in S}=E(x), \\
& \left.\frac{\partial}{\partial n} \Delta \varphi(x)\right|_{x \in S}=D(x),\left.\quad \frac{\partial \varphi(x)}{\partial n}\right|_{x \in S}=F(x), \\
& \text { and } \quad h=\frac{\Gamma[(d-4+s) / 2]}{\pi^{(4-s) / 2} 2^{4-s} \Gamma[(4-s) / 2]}, \quad g=\frac{(s+d-4)(s-2) \Gamma[(d-4+s) / 2]}{\pi^{(4-s) / 2} 2^{4-s} \Gamma[(4-s) / 2]} .
\end{aligned}
$$


It is seen from Eq. (A9) that the fractional biharmonic operator $I_{d}^{4-s}[\Delta \Delta \varphi(x)]$ is equal to the fractional biharmonic operator $\Delta \Delta\left[I_{d}^{4-s} \varphi(x)\right]$ augmented with the boundary integral, which is a parallel to the fractional time derivatives in the Caputo sense relative to that in the Riemann-Liouville sense. It is worth mentioning that the Caputo sense requires $\varphi(x)$ to be 4th-order differentiable. Considering the characteristics of the two different types of definitions, both the fractional Laplacian and the fractional biharmonic operators in the Caputo sense are used in this paper.

Combining definition $\mathrm{A}$ and definition $\mathrm{B}$, a new definition of the space-fractional derivative $(-\Delta)^{s / 2}(0<s \leq 4)$ by a piecewise function is proposed.

\section{b. Definition D}

Fractional Laplacian and fractional biharmonic operators:

$$
(-\Delta)^{s / 2} \varphi(x)= \begin{cases}(-\Delta)_{*}^{s / 2} \varphi(x), & 0<s \leq 2 \\ (-\Delta)_{* *}^{s / 2} \varphi(x), & 2<s \leq 4\end{cases}
$$

where the operator $(-\Delta)_{*}^{s / 2}$ and $(-\Delta)_{* *}^{s / 2}$ are defined by Eqs. (A3) and (A5). Under sufficiently good conditions, the definition satisfies the following Fourier transform ${ }^{38}$

$$
\begin{aligned}
& F\left\{(-\Delta)^{s / 2} \varphi(x), k\right\}=k^{s} \Phi(k), \quad 0<s \leq 4, \\
& (-\Delta)^{s / 2} \varphi(x)=F^{-1}\left\{k^{s} \Phi(k)\right\}=\frac{1}{2 \pi} \int \Phi(k) k^{s} e^{i k x} d k
\end{aligned}
$$

where $k$ is the spatial wave number.

${ }^{1}$ T. L. Szabo, "Diagnostic ultrasound imaging: Inside out," Biomedical Engineering (Academic Press), pp. 297-336.

${ }^{2}$ F. T. D'Astous and F. S. Foster, "Frequency dependence of ultrasound attenuation and backscatter in breast tissue," Ultrasound Med. Biol. 12, 795-808 (1986).

${ }^{3}$ J. Liu and X. C. Wei, "An analysis of seismic scattering attenuation in a random elastic medium," Appl. Geophys. 8, 344-354 (2011).

${ }^{4}$ V. N. Kozlov, A. A. Samokrutov, and V. G. Shevaldykin, "Thickness measurements and flaw detection in concrete using ultrasonic echo method," Nondestr. Test. Eval. 13, 73-84 (1997).

${ }^{5} \mathrm{~F}$. Schubert and B. Köehler, "Three-dimensional time domain modeling of ultrasonic wave propagation in concrete in explicit consideration of aggregates and porosity," J. Comp. Acoust. 9, 1543-1560 (2001).

${ }^{6}$ H. L. Zhang, Theoretical Acoustics (Higher Education Press, China, 2007), pp. 21-27 (in Chinese).

${ }^{7}$ E. N. Landis and S. P. Shah, "Frequency-dependent stress wave attenuation in cement-based materials," Eng. Mech. 121, 737-743 (1995).

${ }^{8}$ R. S. Wu and K. Aki, "The fractal nature of the inhomogeneities in the lithosphere evidenced from seismic wave scattering," Appl. Geophys. 123, 805-818 (1985).

${ }^{9}$ D. P. Blair, "Estimates of seismic attenuation using vibrational resonance and pulse transmission in four large blocks of rock," Geophys. J. Int. 126, 135-146 (1996).

${ }^{10}$ M. N. Toksoz, D. H. Johnston, and A. Timur, "Attenuation of seismic waves in dry and saturated rocks: I. Laboratory measurements," Geophysics 44, 681-690 (1979).

${ }^{11}$ A. Vary, "Material property characterization," Nondestructive Testing Handbook, 2nd ed., Ultrasonic Testing (American Society for Nondestructive Testing, Columbus, OH, 1991), Vol. 7, pp. 383-431.

${ }^{12}$ G. Mavko, T. Mukerji, and J. Dvorkin, The Rock Physics Handbook, Second Edition: Tools for Seismic Analysis of Porous Media (Cambridge University Press, New York, 2009), pp, 150-154.
${ }^{13} \mathrm{~K}$. Aki, "Scattering and attenuation of shear waves in the lithosphere," J. Geophys. Res. 85, 6496-6504, doi:10.1029/JB085iB11p06496 (1980).

${ }^{14}$ D. P. Blair, "A direct comparison between vibrational resonance and pulse transmission data for assessment of seismic attenuation in rock," Geophysics 55, 51-60 (1990a).

${ }^{15}$ M. M. Meerschaert, D. A. Benson, H. P. Scheffler, and B. Baeumer, "Stochastic solution of space-time fractional diffusion equations," Phys. Rev. E 65, 041103 (2002).

${ }^{16} \mathrm{R}$. Metzler and J. Klafter, "The random walk's guide to anomalous diffusion: A fractional dynamics approach,” Phys. Rep. 339, 1-77 (2000).

${ }^{17}$ R. L. Bagley, "Power law and fractional calculus model of viscoelasticity," AIAA J. 27, 1412-1417 (1987).

${ }^{18}$ W. Chen and S. Holm, "Modified Szabos wave equation models for lossy media obeying frequency power law," J. Acoust. Soc. Am. 114, 2570-2574 (2003).

${ }^{19} \mathrm{~W}$. Chen and S. Holm, "Fractional Laplacian time-space models for linear and nonlinear lossy media exhibiting arbitrary frequency power-law dependency," J. Acoust. Soc. Am. 115, 1424-1230 (2004).

${ }^{20}$ J. T. Machadoa, V. Kiryakovab, and F. Mainardi, "Recent history of fractional calculus," Commun. Nonlinear Sci. Numer. Simul. 16, 1140-1153 (2011).

${ }^{21}$ T. L. Szabo, "Time domain wave equations for lossy media obeying a frequency power law," J. Acoust. Soc. Am. 96, 491-500 (1994).

${ }^{22}$ J. F. Kelly, R. J. McGough, and M. M. Meerschaert, "Analytical time domain Green's functions for power-law media,” J. Acoust. Soc. Am. 124, 2861-2872 (2008).

${ }^{23} \mathrm{M}$. Ochmann and S. Makarov, "Representation of the absorption of nonlinear waves by fractional derivative," J. Acoust. Soc. Am. 94, 3392-3399 (1993).

${ }^{24} \mathrm{~B}$. E. Treeby and B. Cox, "Modeling power law absorption and dispersion for acoustic propagation using the fractional Laplacian," J. Acoust. Soc. Am. 127, 2741-2748 (2010).

${ }^{25}$ S. Holm and R. Sinkus, "A unifying fractional wave equation for compressional and shear waves," J. Acoust. Soc. Am 127, 542-548 (2010).

${ }^{26} \mathrm{~S}$. Holm and S. P. Näsholm, "A causal and fractional all-frequency wave equation for lossy media," J. Acoust. Soc. Am. 130, 2195-2202 (2011).

${ }^{27}$ A. D. Pierce, "Acoustics: An introduction to its physical principles and applications," Phys. Today 34(12), 56-57 (1981).

${ }^{28}$ J. C. Bamber, Attenuation and Absorption (Wiley, Chichester, UK, 2005), Chap. 4, pp. 93-166.

${ }^{29}$ S. A. Lambert, S. P. Näsholm, D. Nordsletten, C. Michler, L. Juge, J. M. Serfaty, L. Bilston, B. Guzina, S. Holm, and R. Sinkus, "Bridging three orders of magnitude: Multiple scattered waves sense fractal microscopic structures via dispersion," Phys. Rev. Lett. 115, 094301 (2015).

${ }^{30} \mathrm{P}$. He, "Simulation of ultrasound pulse propagation in lossy media obeying a frequency power law," IEEE Trans. Ultrason. Ferroelectr. Freq. Control 45, 114-125 (1998).

${ }^{31}$ G. F. Pang, "Space-fractional calculus viscoelastic constitutive models for describing non-local acoustic wave dissipation and vibration damping," in Doctoral Dissertation of Hohai University, Nanjing, pp. 68-71, 2015 (in Chinese).

${ }^{32}$ W. C. Horton, Sr., "Dispersion relationships in sediments and sea water," J. Acoust. Soc. Am. 55(3), 547-549 (1974).

${ }^{33}$ W. C. Horton, Sr., Comment on "Kramers-Kronig relationship between ultrasonic attenuation and phase velocity," J. Acoust. Soc. Am. 70, 1182 (1981).

${ }^{34}$ K. R. Waters, M. S. Hughes, J. Mobley, G. H. Brandenburger, and J. G. Miller, "On the applicability of Kramers-Kronig relations for ultrasonic attenuation obeying a frequency power law," J. Acoust. Soc. Am. 108(2), $556-563$ (2000).

${ }^{35}$ H. M. Nussenzveig, Causality and Dispersion Relations, 1 st ed. (Academic, New York, 1972), pp. 3-53.

${ }^{36} \mathrm{M}$. G. Wismer, "Finite element analysis of broadband acoustic pulses through inhomogenous media with power law attenuation," J. Acoust. Soc. Am. 120, 3493-3502 (2006).

${ }^{37} \mathrm{M}$. Caputo, "Linear models of dissipation whose Q is almost frequency independent-II," Geophys. J. Int. 13, 529-539 (1967).

${ }^{38}$ S. Holm, S. P. Näsholm, F. Prieur, and R. Sinkus, "Deriving fractional acoustic wave equations from mechanical and thermal constitutive equations," Comput. Math. Appl. 66(5), 621-629 (2013). 
${ }^{39}$ I. Podlubny, Fractional Differential Equations (Academic, London, 1999), pp. 134-142.

${ }^{40}$ R. L. Bagley and P. J. Torvik, "Fractional calculus-a different approach to the analysis of viscoelastically damped structures," AIAA J. 21, 741-748 (1983).

${ }^{41}$ R. Gorenflo and F. Mainardi, "Random walk models for space-fractional diffusion processes," Fract. Calc. Appl. Anal. 1, 167-191 (1998).

${ }^{42}$ A. A. Kilbas, H. M. Srivastava, and J. J. Trujillo, "Theory and applications of fractional differential equations," North-Holland Math. Stud. 204, 69-133 (2006).
${ }^{43}$ S. G. Samko, A. A. Kilbas, and O. I. Marichev, "Fractional integrals and derivatives: Theory and applications," in Gordon and Breach Science (Gordon and Breach Science Publishers, New York, 1987), pp. $125-130$.

${ }^{44}$ M. Zähle, "Fractional differentiation in the self-affine case. V-The local degree of differentiability," Math. Nachr. 185, 279-306 (1997).

${ }^{45} \mathrm{M}$. J. Buckingham, "Wave-speed dispersion associated with an attenuation obeying a frequency power law," J. Acoust. Soc. Am. 138(5), 2871-2884 (2015). 